\title{
Experimental study of the thermodynamic uncertainty relation
}

\author{
Soham Pal, ${ }^{1}$ Sushant Saryal, ${ }^{1}$ Dvira Segal $\odot,{ }^{2,3}$ T. S. Mahesh, ${ }^{1}$ and Bijay Kumar Agarwalla ${ }^{1, *}$ \\ ${ }^{1}$ Department of Physics, Indian Institute of Science Education and Research, Pune 411008, India \\ ${ }^{2}$ Department of Chemistry, University of Toronto, Toronto, Ontario, Canada M5S $3 \mathrm{H} 6$ \\ ${ }^{3}$ Department of Physics, University of Toronto, Toronto, Ontario, Canada M5S 1A7
}

(Received 19 December 2019; revised manuscript received 14 March 2020; accepted 17 April 2020; published 20 May 2020)

\begin{abstract}
A cost-precision trade-off relationship, the so-called thermodynamic uncertainty relation (TUR), has been recently discovered in stochastic thermodynamics. It bounds certain thermodynamic observables in terms of the associated entropy production. In this Rapid Communication, we experimentally study the TUR in a two-qubit system using an NMR setup. Each qubit is prepared in an equilibrium state, but at different temperatures. The qubits are then coupled, allowing energy exchange (in the form of heat). Using the quantum state tomography technique we obtain the moments of heat exchange within a certain time interval and analyze the relative uncertainty of the energy exchange process. We find that generalized versions of the TUR, which are based on the fluctuation relation, are obeyed. However, the specialized TUR, a tighter bound that is valid under specific dynamics, is violated in certain regimes of operation, in excellent agreement with analytic results. Altogether, this experiment-theory study provides a deep understanding of heat exchange in quantum systems, revealing favorable noise-dissipation regimes of operation.
\end{abstract}

DOI: 10.1103/PhysRevResearch.2.022044

Introduction. Obtaining universal bounds of experimentally accessible physical observables has been a fundamental topic in physics. Such bounds include the Heisenberg uncertainty relation of quantum mechanics, the Carnot bound for the efficiency of heat engines, and the Landauer erasure principle stemming from the second law of thermodynamics. Likewise, recent studies have shown that for systems that are out of equilibrium, there exist trade-off relations between the relative uncertainty of integrated currents (heat, charge) and the associated entropy production [1-42]. These results are now collectively referred to as thermodynamic uncertainty relations (TURs). The specialized version of the TUR (STUR) reads

$$
\frac{\left\langle Q^{2}\right\rangle_{c}}{\langle Q\rangle^{2}} \geqslant \frac{2}{\langle\Sigma\rangle},
$$

where $Q$ represents any integrated current, such as heat or charge, and it is a stochastic variable. $\langle Q\rangle,\left\langle Q^{2}\right\rangle_{c}$ are the average integrated current and its noise, respectively, and $\langle\Sigma\rangle$ is the net average entropy production in the heat exchange process, characterizing irreversibility, or how far the system is driven away from equilibrium. The S-TUR was first conjectured for a continuous-time, discrete-state Markov process in the steady state [1]. It was later proved with the large deviation technique $[2,6]$. Since then, this

\footnotetext{
*bijay@iiserpune.ac.in

Published by the American Physical Society under the terms of the Creative Commons Attribution 4.0 International license. Further distribution of this work must maintain attribution to the author(s) and the published article's title, journal citation, and DOI.
}

relation has been generalized to discrete-time, discretestate Markov processes [8], finite-time statistics [6,7,16,17], Langevin dynamics $[5,16,26,28,32]$, periodically driven systems [20,24], multidimensional systems [16], molecular motors [9], biochemical oscillations [11], interacting oscillators [12], run-and-tumble processes [13], measurement and feedback control $[19,22]$, broken time-reversal symmetry systems $[19,21,23,31,33]$, first-passage times [14,15], and quantum transport problems [34-38,41]. Tighter bounds have also been reported for some stochastic currents [3].

More recently, following the fundamental nonequilibrium fluctuation relation [25], a generalized version of the TUR (G-TUR1) was derived, where the right-hand side of Eq. (1) was modified to $\frac{\left\langle Q^{2}\right\rangle_{c}}{\langle Q\rangle^{2}} \geqslant \frac{2}{\exp \langle\Sigma\rangle-1}$, which is a looser bound compared to Eq. (1). In fact, a more tighter version of the generalized bound had been obtained following a slightly different approach by Timpanaro et al. [39] as $\frac{\left\langle Q^{2}\right\rangle_{c}}{\langle Q\rangle^{2}} \geqslant f(\langle\Sigma\rangle)$, where $f(x)=\operatorname{csch}^{2}[g(x / 2)]$ and $g(x)$ is the inverse function of $x \tanh (x)$. We refer to this bound as the G-TUR2. Interestingly, in the small dissipation limit, $\langle\Sigma\rangle \rightarrow 0$, both these generalized bounds reduce to the S-TUR of Eq. (1). Other weak generalized bounds resulting from the fluctuation theorem were discussed in Ref. [40].

Even with the discovery of the G-TUR, there are compelling reasons to continue and investigate the S-TUR. (i) First and foremost, since the S-TUR is a tighter bound than the G-TUR, once satisfied it offers more definite information on performance. (ii) The S-TUR was proved for different classes of models, in particular, discrete-state Markov processes. However, several quantum transport models [35-37] illustrate its validity in certain parameter regimes-albeit the underlying quantum dynamics cannot be simply uniquely classified 
by its degree of Markovianity (for quantum systems, there is no agreement on the definition of non-Markovianity [43]). (iii) Notably, the S-TUR can be assessed from a fundamental nonequilibrium viewpoint, by studying its perturbative expansion, with the equilibrium value as the reference point $[35,36]$. This series expansion approach does not rely on the notion of Markovianity, thus it allows a broader perspective on the validity of the S-TUR.

Specifically, for a heat exchange problem in a steady state the cumulants can be expanded close to equilibrium in terms of the thermal affinity $\Delta \beta=\beta_{1}-\beta_{2}$ around a fixed inverse temperature $\beta$,

$$
\begin{aligned}
\langle Q\rangle & =G_{1} \Delta \beta+G_{2} \frac{(\Delta \beta)^{2}}{2 !}+G_{3} \frac{(\Delta \beta)^{3}}{3 !}+\cdots, \\
\left\langle Q^{2}\right\rangle_{c} & =S_{0}+S_{1} \Delta \beta+S_{2} \frac{(\Delta \beta)^{2}}{2 !}+\cdots, \\
\left\langle Q^{3}\right\rangle_{c} & =R_{1} \Delta \beta+\cdots .
\end{aligned}
$$

Here, $G_{1}$ is the linear transport coefficient and $S_{0}$ is the equilibrium noise. $G_{2}, G_{3}, \ldots\left(S_{1}, S_{2}, \ldots\right)$ are higher-order nonequilibrium transport (noise) coefficients. As a consequence of the exact fluctuation symmetry, the following relations hold [44]: $S_{0}=2 G_{1}, S_{1}=G_{2}, 3 S_{2}-2 G_{3}=R_{1}$, and so on. This leads to [36] $(\langle\Sigma\rangle=\Delta \beta\langle Q\rangle)$,

$$
\langle\Sigma\rangle \frac{\left\langle Q^{2}\right\rangle_{c}}{\langle Q\rangle^{2}}=2+\frac{(\Delta \beta)^{2}}{6} \frac{R_{1}}{G_{1}}+O(\Delta \beta)^{3} .
$$

While the linear coefficient for the average heat exchange $G_{1}$ is always positive, the skewness $R_{1}$ does not take a definite sign; when $R_{1} \geqslant 0$, the S-TUR is valid to that order, while $R_{1}<0$ indicates $\mathrm{S}$-TUR violations.

In this Rapid Communication, we examine experimentally and analytically the S-TUR based on the perturbative expansion (3), beyond the classical, Markovian scenario. Despite intense theoretical efforts dedicated to derive and analyze the TUR, experimental studies of this trade-off relation are still limited to a kinetic-network analysis of biological molecular motors [9] and charge transport in atomic-scale junctions [10]. Nevertheless, both studies are concerned with problems that obey the S-TUR.

We focus on the problem of quantum heat exchange between two initially thermalized qubits in a NMR setup, in the transient regime. Moments of heat exchange are obtained by performing quantum state tomography (QST) for the qubits. As expected, G-TURs are valid throughout. This agreement, while fundamentally important, does not offer any practical input for system performance or in the design of quantum heat machines. More interestingly, we identify regimes of validity for the S-TUR in this quantum system, and quantify its violation, thus pinpointing favorable regimes of operation, with an excellent agreement between theory and measurements.

Cumulants of heat exchange. Consider two systems with their Hamiltonians $H_{1}$ and $H_{2}$ that are initially $(t<0)$ decoupled and separately prepared at their respective thermal equilibrium state. The initial composite density matrix is thus given as a product state, $\rho(0)=\rho_{1} \otimes \rho_{2}$, with $\rho_{i}=$ $\exp \left[-\beta_{i} H_{i}\right] / \mathcal{Z}_{i}, i=1,2$, the Gibbs thermal state with inverse temperature $\beta_{i}=1 / k_{B} T_{i}$ ( $k_{B}$ is the Boltzmann constant) and $\mathcal{Z}_{i}=\operatorname{Tr}\left[e^{-\beta_{i} H_{i}}\right]$ the corresponding equilibrium partition function. The coupling between the systems is suddenly switched on at $t=0$ for a duration $\tau$ (total Hamiltonian $\mathcal{H}$ ), which allows energy exchange between the two systems. Due to the randomness of the initial thermal state and the inherent probabilistic nature of quantum mechanics, the exchanged energy is not a deterministic quantity, but rather quantified with a probability distribution function (PDF). In the quantum regime, this PDF is constructed by following a two-point projective measurement scheme [45-47]: The first projective measurement of the energy of the two systems is performed before they are coupled. A second projective measurement is done at the end of the energy exchange process (after the systems are separated). This procedure respects the fundamental Jarzynski and Wöjcik exchange fluctuation symmetry [48]. For the bipartite setup considered here, the joint PDF corresponding to energy change $\left(\Delta E_{i}, i=1,2\right)$ between the systems, during a coupling interval $\tau$, is denoted by $p_{\tau}\left(\Delta E_{1}, \Delta E_{2}\right)$. It can be shown that $[49,50]$

$$
\begin{aligned}
& \left\langle\left(e^{-\beta_{1} \Delta E_{1}-\beta_{2} \Delta E_{2}}\right)^{z}\right\rangle_{\tau} \\
& \quad=\int d\left(\Delta E_{1}\right) d\left(\Delta E_{2}\right) p_{\tau}\left(\Delta E_{1}, \Delta E_{2}\right) e^{-z \beta_{1} \Delta E_{1}-z \beta_{2} \Delta E_{2}} \\
& \quad=\operatorname{Tr}\left[\rho(0)^{z} \rho(\tau)^{1-z}\right],
\end{aligned}
$$

with $\rho(0)$ the combined density matrix of the two systems at the moment they are coupled, and $\rho(\tau)$ their density matrix at the end of their coupled evolution. We now consider the case $\Delta E_{1} \approx-\Delta E_{2}$, which is justified when the two systems are only weakly coupled. Alternatively, this approximation becomes an exact equality if there is no energy cost involved in turning on and off the interaction between the two systems. Interpreting the energy change for individual systems as heat, $\Delta E_{1}=-\Delta E_{2}=Q$, we directly get from Eq. (4) an expression for the moments of heat exchange [50],

$$
\left\langle Q^{n}\right\rangle_{\tau}=\frac{1}{(\Delta \beta)^{n}} \operatorname{Tr}\left\{\rho(\tau) \mathrm{T}_{n}[\ln \rho(\tau)-\ln \rho(0)]^{n}\right\},
$$

where $n=1,2, \ldots$ corresponds to the order of the heat exchange moment and $\Delta \beta=\beta_{1}-\beta_{2} . \mathrm{T}_{n}$ is the time-ordering operator; it places operators at the latest time to the left. This powerful expression offers a unique way to gather moments of heat exchange, simply by performing quantum state tomography based on NMR experiments. Alternatively, cumulants of heat exchange can be obtained by implementing an ancilla-based interferometric technique [51-54]. This method gives direct access to the characteristic function (CF) of heat [55,56], defined using the two-point measurement protocol,

$$
\begin{aligned}
\chi_{\tau}(u) & =\int d Q e^{i u Q} p_{\tau}(Q) \\
& =\operatorname{Tr}\left[\mathcal{U}^{\dagger}(\tau, 0)\left(e^{i u H_{1}} \otimes 1_{2}\right) \mathcal{U}(\tau, 0)\left(e^{-i u H_{1}} \otimes 1_{2}\right) \rho(0)\right] .
\end{aligned}
$$

Here, $u$ is the variable conjugate to $Q$, and $\mathcal{U}(t, 0)=e^{-i \mathcal{H} t / \hbar}$ is the unitary propagator with the total Hamiltonian $\mathcal{H}$. In the language of the $\mathrm{CF}$, the exchange fluctuation symmetry translates to $\chi_{\tau}(u)=\chi_{\tau}(-u+i \Delta \beta)[48,56-59]$.

Theoretical analysis. We now describe a specific case, the so-called $X Y$ model consisting of two qubits with the 
Hamiltonian

$$
\begin{aligned}
\mathcal{H}_{X Y}= & \frac{h v_{0}}{2} \sigma_{1}^{z} \otimes 1_{2}+1_{1} \otimes \frac{h v_{0}}{2} \sigma_{2}^{z} \\
& +\frac{h J}{2}\left(\sigma_{1}^{x} \otimes \sigma_{2}^{y}-\sigma_{1}^{y} \otimes \sigma_{2}^{x}\right) .
\end{aligned}
$$

Here, $H_{1}=\frac{h v_{0}}{2} \sigma_{1}^{z} \otimes 1_{2}, H_{2}=1_{1} \otimes \frac{h \nu_{0}}{2} \sigma_{2}^{z}$, with $v_{0}$ the frequency of the qubits, and $\sigma_{i}, i=x, y, z$, are the standard Pauli matrices. The last term, denoted by $H_{12}$, represents the interaction between the qubits, with $J$ the coupling parameter. An important feature of this model is that $\left[H_{12}, H_{1}+H_{2}\right]=0$. This commutation implies that the change of energy for one qubit is exactly compensated by the other qubit, as there is no energy cost involved in turning on or off the interaction between the qubits. For such an "energy-preserving" Hamiltonian, $\Delta E_{1}=-\Delta E_{2}=Q$ is exact and the average entropy production simply reduces to $\langle\Sigma\rangle=\left(\beta_{1}-\beta_{2}\right)\langle Q\rangle$.

Cumulants of heat exchange can either be computed from the composite density matrix [50], or directly from the CF $\chi_{\tau}(u)$ of heat, following Eq. (6). We take the latter approach for the $X Y$ model; algebraic manipulations of the Pauli matrices yield [54]

$$
\begin{aligned}
\chi_{\tau}(u)= & {\left[1+\sin ^{2}(2 \pi J \tau)\left\{f_{1}\left(v_{0}\right)\left[1-f_{2}\left(v_{0}\right)\right]\left(e^{-i h u v_{0}}-1\right)\right.\right.} \\
& \left.\left.+f_{2}\left(v_{0}\right)\left[1-f_{1}\left(v_{0}\right)\right]\left(e^{i h u v_{0}}-1\right)\right\}\right],
\end{aligned}
$$

where $f_{i}\left(v_{0}\right)=\left(e^{\beta_{i} h v_{0}}+1\right)^{-1}, i=1,2$. For compactness, below we identify these functions as $f_{1,2}$. It is easy to verify that the above $\mathrm{CF}$ satisfies the exchange fluctuation symmetry for arbitrary values of $J, \tau, \beta_{1}, \beta_{2}$, and $\nu_{0}$. Expressions for the average heat current and the associated noise are derived by taking successive derivatives of $\ln \chi_{\tau}(u)$ with respect to $i u$. We write down the first three cumulants, useful for the analysis of the TUR,

$$
\begin{aligned}
\langle Q\rangle_{\tau}= & h v_{0} \mathcal{T}_{\tau}(J)\left[f_{2}-f_{1}\right] \\
\left\langle Q^{2}\right\rangle_{\tau}^{c}= & \left(h v_{0}\right)^{2}\left[\mathcal{T}_{\tau}(J)\left[f_{1}\left(1-f_{2}\right)+f_{2}\left(1-f_{1}\right)\right]\right. \\
& \left.-\mathcal{T}_{\tau}^{2}(J)\left(f_{2}-f_{1}\right)^{2}\right] \\
\left\langle Q^{3}\right\rangle_{\tau}^{c}= & \left(h v_{0}\right)^{3} \mathcal{T}_{\tau}(J)\left(f_{2}-f_{1}\right)\left[1-3 \mathcal{T}_{\tau}(J)\left(f_{1}\left(1-f_{2}\right)\right.\right. \\
& \left.\left.+\left(1-f_{1}\right) f_{2}\right)+2 \mathcal{T}_{\tau}^{2}(J)\left(f_{1}-f_{2}\right)^{2}\right]
\end{aligned}
$$

Here, $\mathcal{T}_{\tau}(J)=\sin ^{2}(2 \pi J \tau)$.

In the weak-coupling limit. i.e., $J \tau \ll 1, \mathcal{T}_{\tau}^{2}(J) \ll \mathcal{T}_{\tau}(J)$, the S-TUR bound is satisfied, even far from equilibrium [60]. This weak-coupling limit corresponds to a Poisson process, missing cotunneling energy transfer processes, which are quadratic in the transmission coefficient $\mathcal{T}_{\tau}^{2}(J)$. Since the tight S-TUR bound is valid in our model at weak coupling, it is meaningful to examine it beyond this regime and identify its violations, which essentially correspond to non-Markovianity.

To identify and quantify S-TUR violations, we turn to the perturbative expansion (3), which was derived for steady state transport [35]. However, since in the present model the role of the coupling time is simply to scale the interaction strength, the exchange fluctuation symmetry holds for arbitrary time $\tau$, and as a result, Eq. (3) is valid - albeit with time-dependent cumulants, $G_{1}(\tau), S_{0}(\tau), G_{2}(\tau), G_{3}(\tau), \ldots$ Specifically, for (a)

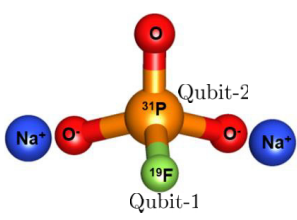

Sodium fluorophosphate

(b)

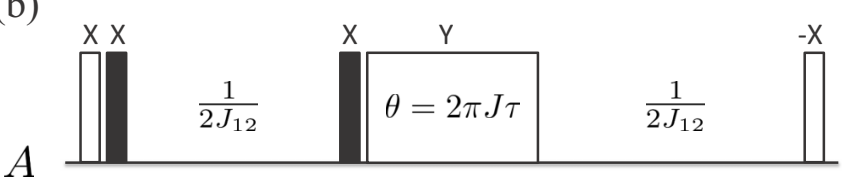

$B$

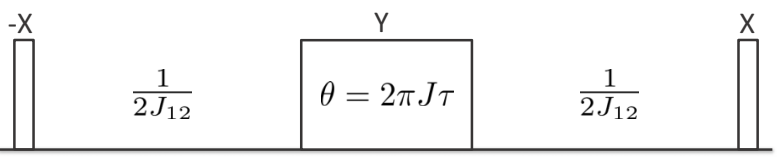

FIG. 1. (a) Molecular structure of the two-qubit NMR spin system, sodium fluorophosphate. The NMR active spin- $1 / 2,{ }^{19} \mathrm{~F}$ and ${ }^{31} \mathrm{P}$ nuclei in the molecule, labeled as qubit 1 and qubit 2, respectively, are coupled by the Hamiltonian (11) with the coupling strength $J_{12}=868 \mathrm{~Hz}$. (b) Pulse sequence to realize heat exchange coupling Hamiltonian $\mathcal{H}_{X Y}$ in Eq. (6). The pulses are applied on qubits 1 and 2 in a time ordered manner from left to right. The black and white narrow solid bars represent $\pi$ and $\pi / 2$ pulses, respectively, with the phases mentioned above them. $1 / 2 J_{12}$ represents the free evolution delay. The white box represents the $\theta$ (in rad) angle pulse about the $y$ axis.

the $X Y$ model, we get $\left[f\left(v_{0}\right)\right.$ is evaluated at $\left.\beta\right]$,

$$
\begin{aligned}
& G_{1}(\tau)=\left(h v_{0}\right)^{2} \mathcal{T}_{\tau}(J) f(1-f) \geqslant 0, \\
& R_{1}(\tau)=\left(h v_{0}\right)^{4} \mathcal{T}_{\tau}(J) f(1-f)\left[1-6 \mathcal{T}_{\tau}(J) f(1-f)\right] .
\end{aligned}
$$

To order $(\Delta \beta)^{2}$, Eq. (3) simplifies to

$$
\Delta \beta \frac{\left\langle Q^{2}\right\rangle_{\tau}^{c}}{\langle Q\rangle_{\tau}}=2+\left(\Delta \beta h \nu_{0}\right)^{2}\left[\frac{1}{6}-\mathcal{T}_{\tau}(J) f(1-f)\right] .
$$

The S-TUR is violated when $R_{1}(\tau)<0$, that is, $\mathcal{T}_{\tau}(J) f(1-$ $f)>1 / 6$. However, since $0 \leqslant f(1-f) \leqslant 1 / 4$, the S-TUR is violated once $\mathcal{T}_{\tau}(J)>\frac{2}{3}$. Interestingly, already in the quadratic order of $\Delta \beta$, the TUR can drop below the value of 2 if $\mathcal{T}_{\tau}(J)$ crosses a critical value. We assess the perturbative formula (10) in Ref. [50]. However, in the weak-coupling limit, i.e., $J \tau \ll 1, \mathcal{T}_{\tau}^{2}(J) \ll \mathcal{T}_{\tau}(J)$, and $R_{1}(\tau)$ is always positive. Moreover, it can be shown that in this limit the S-TUR bound is always above 2, even far from equilibrium [60].

Experimental setup and results. To study heat exchange between two qubits we use liquid-state NMR spectroscopy of the ${ }^{19} \mathrm{~F}$ and ${ }^{31} \mathrm{P}$ nuclei in the molecule sodium fluorophosphate dissolved in $\mathrm{D}_{2} \mathrm{O}$. Experiments are performed in a $500-\mathrm{MHz}$ Bruker NMR spectrometer at ambient temperature. As shown in Fig. 1(a), ${ }^{19} \mathrm{~F}$ and ${ }^{31} \mathrm{P}$ are identified as the two qubits, 1 and 2 , exchanging heat under the desired coupling Hamiltonian, Eq. (6). As the sample is in the liquid state, the molecules can be considered identical with intermolecular interactions averaged out due to motional averaging. All the experimental procedures, initialization of the system and the heat exchange, are completed in timescales much shorter than the relaxation time of the nuclei. The internal Hamiltonian $H_{\text {int }}$ of the 

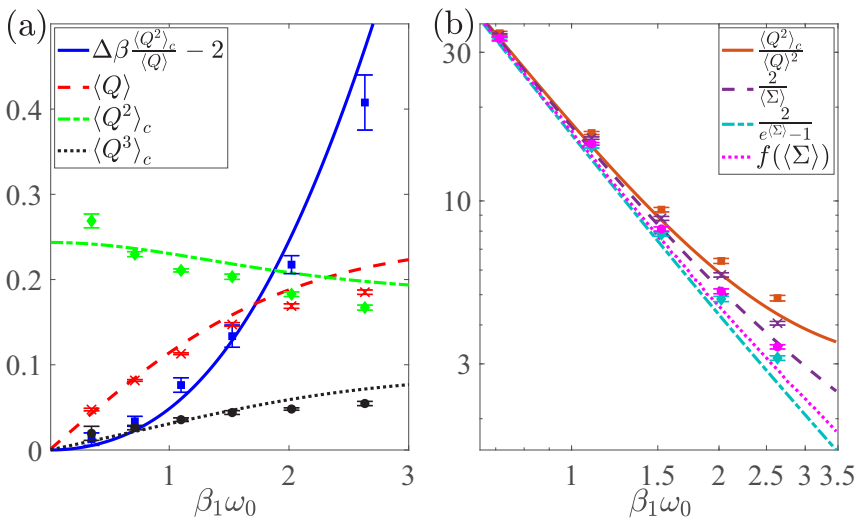

FIG. 2. (a) First three cumulants of heat exchange, along with a measure for the S-TUR, as a function of the inverse temperature of qubit $1 \beta_{1} ; \beta_{2}=0$. Measurements (square, cross, diamond, and circle symbols) are constructed with the help of Eq. (5), and are compared to the theory (solid, dark-dashed, light dashed-dotted, and dotted lines), Eq. (8). (b) Comparison between different bounds, showing that the S-TUR (experiment: cross; theory: dashed line) provides the tightest lower bound to $\frac{\left\langle Q^{2}\right\rangle_{\tau}^{c}}{\langle Q\rangle_{\tau}^{2}}$ (experiment: square; theory: solid line). Experimental results are obtained from state tomography, yielding $\langle Q\rangle_{\tau}$, which is used to calculate the entropy production. Theoretical results are based on Eq. (8). Parameters are $J \tau=1 / 8$ and $\nu_{0}=\pi / 20$ $\left(\omega_{0}=2 \pi v_{0}\right)$. Error bars are obtained by repeating the experiments eight times.

two spins-in the rotating frame of the radio-frequency (rf) pulses-can be written as

$$
H_{\text {int }}=\frac{\pi}{2} J_{12} \sigma_{1}^{z} \sigma_{2}^{z},
$$

where $J_{12}=868 \mathrm{~Hz}$ is the scalar coupling between the ${ }^{19} \mathrm{~F}$ and ${ }^{31} \mathrm{P}$ nuclei, as explained in Fig. 1(a). The desired coupling Hamiltonian $\mathcal{H}_{X Y}$ under which the spins exchange heat is realized from the internal Hamiltonian $H_{\text {int }}$ with the rf pulses displayed in Fig. 1(b). The net effect of the pulse sequence is that the two spins evolve under the coupling Hamiltonian $\mathcal{H}_{X Y}$ for a duration $\tau$ that is specified by the $\theta$ angle rotation about the $y$ axis, as shown. For the duration of $1 /\left(2 J_{12}\right)$, the system evolves under the Hamiltonian $H_{\text {int }}$.

To start with, the two qubits are initialized in a pseudoequilibrium state $\rho_{1} \otimes \rho_{2}$, where $\rho_{i}=\exp \left[-\beta_{i} H_{i}\right] / \mathcal{Z}_{i}$ is a Gibbs thermal state with inverse pseudospin temperatures $\beta_{i}$ and $\mathcal{Z}_{i}$ the partition function. For simplicity, we set $\beta_{2}=0$ in all our measurements. Qubit 1 is prepared at a higher inverse temperature $\beta_{1}$ by initializing it in a pseudopure state (PPS) of $|0\rangle\langle 0|$, followed by applying pulses between 0 and $\pi / 2$, and a pulse field gradient (PFG). The purpose of the PFG is to destroy coherences produced by 0 to $\pi / 2$ angle pulses. The qubits - prepared at two different pseudoequilibrium statesare made to exchange heat under the coupling Hamiltonian $\mathcal{H}_{X Y}$ for different time intervals $\tau$ and different $\beta_{1}$. Following the coupling period, we perform QST of the final state (in addition to the QST of the initial pseudoequilibrium state) [50], and from Eq. (5) achieve the cumulants of heat exchange.

In Figs. 2 and 3 we present two cases, displaying agreement and violation, respectively, of the S-TUR. First, in Fig. 2, we set $J \tau=1 / 8$. According to the theoretical analysis, the
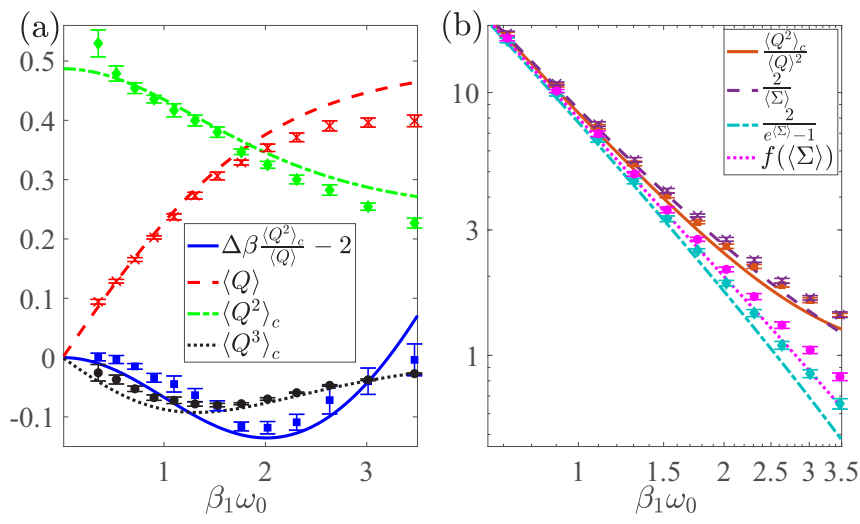

FIG. 3. Same as Fig. 2 but at $J \tau=1 / 4$ leading to $\mathcal{T}_{\tau}(J)>2 / 3$, therefore the violation of the S-TUR.

S-TUR is valid (to the lowest perturabtive order) when the skewness is positive, or $\mathcal{T}_{\tau}(J)=1 / 2<2 / 3$. Indeed, we find in Fig. 2(a) that both $R_{1}(\tau)$ and $\Delta \beta \frac{\left\langle Q^{2}\right\rangle_{\tau}^{c}}{\langle Q\rangle_{\tau}}-2$ are positive for all $\Delta \beta$. In Fig. 2(b), we compare the different bounds on the relative uncertainty $\frac{\left\langle Q^{2}\right\rangle_{\tau}^{c}}{\langle Q\rangle_{\tau}^{2}}$, using experimental data as well as theoretically, and show that the S-TUR provides the tightest bound. Next, in Fig. 3(a) we display results for $J \tau=1 / 4$, for which, according to our theory, violations of the S-TUR are expected to occur already in the quadratic order of $\Delta \beta$, as $\mathcal{T}_{\tau}(J)=1>2 / 3$. Indeed, we clearly see a violation for $0<\beta_{1} \omega_{0}<3.2$. Furthermore, the third cumulant, $\left\langle Q^{3}\right\rangle_{c}$, is negative in this region, which corroborates with Eq. (10). The theoretically predicted lowest value for the S-TUR for this model is $\Delta \beta \frac{\left\langle Q^{2}\right\rangle_{\tau}^{c}}{\langle Q\rangle_{\tau}} \approx 1.86$, and we experimentally reach a value very close to this number. The violation of the S-TUR can also be seen in Fig. 3(b): The S-TUR bound $(2 /\langle\Sigma\rangle)$ appears above the ratio $\frac{\left\langle Q^{2}\right\rangle_{\tau}^{c}}{\langle Q\rangle_{\tau}^{2}}$, and it is greater than the other,

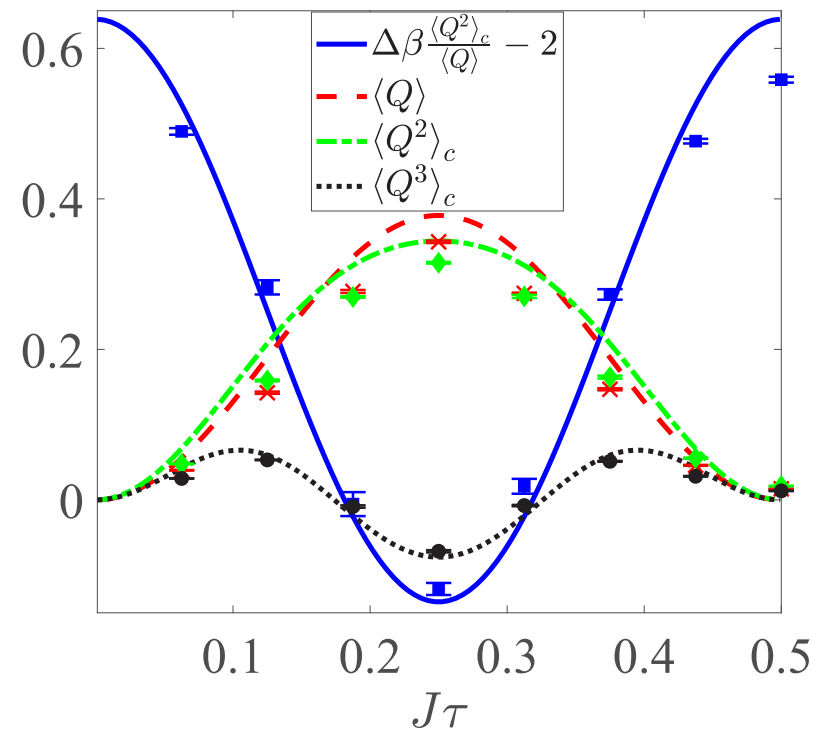

FIG. 4. Cumulants of heat exchange and the S-TUR as a function of $J \tau$ for $J=1 \mathrm{~Hz}, \beta_{1} \omega_{0}=2.02$, and $\beta_{2}=0$. Other parameters are the same as in Fig. 2. 
looser bounds. Measurements again closely match the theoretical curves.

A complete analysis of the TUR as a function of the heat exchange duration $\tau$ and for a fixed $J=1 \mathrm{~Hz}$ is presented in Fig. 4. We display the first three cumulants and note that the relative uncertainty is reduced (violation of S-TUR) within a certain region of parameters: The minimum value of the S-TUR precisely appears when the fluctuations of the heat exchange are reduced, below the value of the first cumulant. As expected, the skewness is found to be negative in this region.

Summary. We experimentally examined the TUR for heat exchange by realizing the $X Y$ model, performing quantum state tomography, and extracting the heat exchange cumulants. We found that the S-TUR provides a tight bound up to a certain threshold value for the qubit-qubit coupling parameter $\sin ^{2}(2 \pi J \tau)$, beyond which the bound is invalidated. As predicted theoretically, the validity of the S-TUR crucially depends on the sign of the third cumulant. Generalized versions of the TUR are satisfied throughout in our system, as expected, since these (weak) bounds are derived from the universal fluctuation relations, which is satisfied in our experimental setup. Nevertheless, a most interesting observation is that the tighter S-bound is in fact also satisfied over a wide range of the coupling value $J \tau$. The S-TUR thus contains practical information: The condition to invalidate it pinpoints to regimes of favorable performance for heat machines, operating with high constancy and little dissipation.

Acknowledgments. B.K.A. gratefully acknowledges the startup funding from IISER Pune and the Max PlanckIndia mobility grant. T.S.M. acknowledges the support from the Department of Science and Technology, India (Grant No. DST/SJF/PSA-03/2012-13) and the Council of Scientific and Industrial Research (CSIR), India [Grant No. CSIR-03(1345)/16/EMR-II]. S.S. acknowledges support from CSIR, India (Grant No. 1061651988). The work of D.S. is supported by the Canada Research Chairs program and an NSERC discovery grant. B.K.A. thanks Gabriel Landi and Gernot Schaller, S.S. thanks Deepak Dhar, and S.P. thanks V. R. Krithika for insightful discussions.
[1] A. C. Barato and U. Seifert, Thermodynamic Uncertainty Relation for Biomolecular Processes, Phys. Rev. Lett. 114, 158101 (2015).

[2] T. R. Gingrich, J. M. Horowitz, N. Perunov, and J. L. England, Dissipation Bounds all Steady State Current Fluctuations, Phys. Rev. Lett. 116, 120601 (2016).

[3] M. Polettini, A. Lazarescu, and M. Esposito, Tightening the uncertainty principle for stochastic currents, Phys. Rev. E 94, 052104 (2016).

[4] P. Pietzonka, A. C. Barato, and U. Seifert, Universal bounds on current fluctuations, Phys. Rev. E 93, 052145 (2016).

[5] C. Hyeon and W. Hwang, Physical insight into the thermodynamic uncertainty relation using Brownian motion in tilted periodic potentials, Phys. Rev. E 96, 012156 (2017).

[6] J. M. Horowitz and T. R. Gingrich, Proof of the finite-time thermodynamic uncertainty relation for steady-state currents, Phys. Rev. E 96, 020103(R) (2017).

[7] S. Pigolotti, I. Neri, E. Roldán, and F. Jülicher, Generic Properties of Stochastic Entropy Production, Phys. Rev. Lett. 119, 140604 (2017).

[8] K. Proesmans and C. V. den Broeck, Discrete-time thermodynamic uncertainty relation, Europhys. Lett. 119, 20001 (2017).

[9] W. Hwang, and C. Hyeon, Energetic costs, precision, and transport efficiency of molecular motors, J. Phys. Chem. Lett. 9, 513 (2018).

[10] H. M. Friedman, B. K. Agarwalla, O. Shein-Lumbroso, O. Tal, and D. Segal, Thermodynamic uncertainty relation in atomicscale quantum conductors, arXiv:2002.00284.

[11] R. Marsland, W. Cui, and J. M. Horowitz, The thermodynamic uncertainty relation in biochemical oscillations, J. Roy. Soc. Interface 16, 20190098 (2019).

[12] S. Lee, C. Hyeon, and J. Jo, Thermodynamic uncertainty relation of interacting oscillators in synchrony, Phys. Rev. E 98, 032119 (2018).
[13] M. Shreshtha and R. J. Harris, Thermodynamic uncertainty for run-and-tumble-type processes, Europhys. Lett. 126, 40007 (2019).

[14] J. P. Garrahan, Simple bounds on fluctuations and uncertainty relations for first-passage times of counting observables, Phys. Rev. E 95, 032134 (2017).

[15] T. R. Gingrich and J. M. Horowitz, Fundamental Bounds on First Passage Time Fluctuations for Currents, Phys. Rev. Lett. 119, 170601 (2017).

[16] A. Dechant, Multidimensional thermodynamic uncertainty relations, J. Phys. A: Math. Theor. 52, 035001 (2019).

[17] P. Pietzonka, F. Ritort, and U. Seifert, Finite-time generalization of the thermodynamic uncertainty relation, Phys. Rev. E 96, 012101 (2017).

[18] G. Falasco, M. Esposito, and J.-C. Delvenne, Unifying thermodynamic uncertainty relations, arXiv:1906.11360.

[19] P. P. Potts and P. Samuelsson, Thermodynamic uncertainty relations including measurement and feedback, Phys. Rev. E 100, 052137 (2019).

[20] T. Koyuk, U. Seifert, and P. Pietzonka, A generalization of the thermodynamic uncertainty relation to periodically driven systems, J. Phys. A: Math. Theor. 52, 02LT02 (2018).

[21] K. Macieszczak, K. Brandner, and J. P. Garrahan, Unified Thermodynamic Uncertainty Relations in Linear Response, Phys. Rev. Lett. 121, 130601 (2018).

[22] T. Van Vu and Y. Hasegawa, Uncertainty relation under information measurement and feedback control, J. Phys. A: Math. Theor. 53, 075001 (2020).

[23] K. Proesmans and J. M. Horowitz, Hysteretic thermodynamic uncertainty relation for systems with broken time-reversal symmetry, J. Stat. Mech. (2019) 054005.

[24] A. C. Barato, R. Chetrite, A. Faggionato, and D. Gabrielli, Bounds on current fluctuations in periodically driven systems, New J. Phys. 20, 103023 (2018). 
[25] Y. Hasegawa and T. Van Vu, Fluctuation Theorem Uncertainty Relation, Phys. Rev. Lett. 123, 110602 (2019).

[26] Y. Hasegawa and T. V. Vu, Uncertainty relations in stochastic processes: An information inequality approach, Phys. Rev. E 99, 062126 (2019).

[27] M. L. Rosinberg and G. Tarjus, Comment on thermodynamic uncertainty relation for time-delayed Langevin systems, arXiv: 1810.12467.

[28] T. R. Gingrich, G. M. Rotskoff, and J. M. Horowitz, Inferring dissipation from current fluctuations, J. Phys. A: Math. Theor. 50, 184004 (2017).

[29] A. Dechant and S.-i. Sasa, Current fluctuations and transport efficiency for general Langevin systems, J. Stat. Mech. (2018) 063209.

[30] I. Di Terlizzi and M. Baiesi, Kinetic uncertainty relation, J. Phys. A: Math. Theor. 52, 02 LT03 (2018).

[31] K. Brandner, T. Hanazato, and K. Saito, Thermodynamic Bounds on Precision in Ballistic Multiterminal Transport, Phys. Rev. Lett. 120, 090601 (2018).

[32] D. Gupta and A. Maritan, Thermodynamic uncertainty relations via second law of thermodynamics, Eur. Phys. J. B 93, 28 (2020).

[33] H.-M. Chun, L. P. Fischer, and U. Seifert, Effect of a magnetic field on the thermodynamic uncertainty relation, Phys. Rev. E 99, 042128 (2019).

[34] K. Ptaszynski, Coherence-enhanced constancy of a quantum thermoelectric generator, Phys. Rev. B 98, 085425 (2018).

[35] B. K. Agarwalla and D. Segal, Assessing the validity of the thermodynamic uncertainty relation in quantum systems, Phys. Rev. B 98, 155438 (2018).

[36] S. Saryal, H. Friedman, D. Segal, and B. K. Agarwalla, Thermodynamic uncertainty relation in thermal transport, Phys. Rev. E 100, 042101 (2019)

[37] J. Liu and D. Segal, Thermodynamic uncertainty relation in quantum thermoelectric junctions, Phys. Rev. E 99, 062141 (2019).

[38] S. Kheradsoud, N. Dashti, M. Misiorny, P. P. Potts, J. Splettstoesser, and P. Samuelsson, Power, efficiency and fluctuations in a quantum point contact as steady-state thermoelectric heat engine, Entropy 21, 777 (2019).

[39] A. M. Timpanaro, G. Guarnieri, J. Goold, and G. T. Landi, Thermodynamic Uncertainty Relations from Exchange Fluctuation Theorems, Phys. Rev. Lett. 123, 090604 (2019).

[40] H. Vroylandt, K. Proesmans, and T. R. Gingrich, Isometric uncertainty relations, J. Stat. Phys. 178, 1039 (2020).

[41] G. Guarnieri, G. T. Landi, S. R. Clark, and J. Goold, Thermodynamics of precision in quantum non-equilibrium steady states, Phys. Rev. Res. 1, 033021 (2019).

[42] J. M. Horowitz and T. R. Gingrich, Thermodynamic uncertainty relations constrain non-equilibrium fluctuations, Nat. Phys. 16, 15 (2020).

[43] L. Li, M. J. Hall, and H. M. Wiseman, Concepts of quantum non-Markovianity: A hierarchy, Phys. Rep. 759, 1 (2018).
[44] K. Saito and Y. Utsumi, Symmetry in full counting statistics, fluctuation theorem, and relations among nonlinear transport coefficients in the presence of a magnetic field, Phys. Rev. B 78, 115429 (2008).

[45] M. Esposito, U. Harbola, and S. Mukamel, Nonequilibrium fluctuations, fluctuation theorems, and counting statistics in quantum systems, Rev. Mod. Phys. 81, 1665 (2009).

[46] M. Campisi, P. Hänggi, and P. Talkner, Colloquium: Quantum fluctuation relations: Foundations and applications, Rev. Mod. Phys. 83, 771 (2011).

[47] M. Campisi, P. Talkner, and P. Hänggi, Influence of measurements on the statistics of work performed on a quantum system, Phys. Rev. E 83, 041114 (2011).

[48] C. Jarzynski and D. K. Wójcik, Classical and Quantum Fluctuation Theorems for Heat Exchange, Phys. Rev. Lett. 92, 230602 (2004).

[49] B. B. Wei, Relations between heat exchange and Rényi divergences, Phys. Rev. E 97, 042107 (2018).

[50] See Supplemental Material at http://link.aps.org/supplemental/ 10.1103/PhysRevResearch.2.022044 for details of the derivation for heat exchange moments, further experimental details, and the validity of the perturbative expansion.

[51] L. Mazzola, G. De Chiara, and M. Paternostro, Measuring the Characteristic Function of the Work Distribution, Phys. Rev. Lett. 110, 230602 (2013).

[52] R. Dorner, S. R. Clark, L. Heaney, R. Fazio, J. Goold, and V. Vedral, Extracting Quantum Work Statistics and Fluctuation Theorems by Single-Qubit Interferometry, Phys. Rev. Lett. 110, 230601 (2013).

[53] M. Campisi, R. Blattmann, S. Kohler, D. Zueco, and P. Hanggi, Employing circuit QED to measure non-equilibrium work fluctuations, New J. Phys. 15, 105028 (2013).

[54] S. Pal, T. S. Mahesh, and B. K. Agarwalla, Experimental demonstration of the validity of the quantum heat-exchange fluctuation relation in an NMR setup, Phys. Rev. A 100, 042119 (2019).

[55] G. T. Landi and D. Karevski, Fluctuations of the heat exchanged between two quantum spin chains, Phys. Rev. E 93, 032122 (2016).

[56] B. K. Agarwalla, H. Li, B. Li, and J.-S. Wang, Exchange fluctuation theorem for heat transport between multiterminal harmonic systems, Phys. Rev. E 89, 052101 (2014).

[57] B. K. Agarwalla, B. Li, and J.-S. Wang, Full-counting statistics of heat transport in harmonic junctions: Transient, steady states, and fluctuation theorems, Phys. Rev. E 85, 051142 (2012).

[58] K. Saito and A. Dhar, Fluctuation Theorem in Quantum Heat Conduction, Phys. Rev. Lett. 99, 180601 (2007).

[59] T. Denzler and E. Lutz, Heat distribution of a quantum harmonic oscillator, Phys. Rev. E 98, 052106 (2018).

[60] In the weak-coupling limit, the second cumulant (8) can be organized as [using $x \operatorname{coth}(x) \geqslant 1] \quad\left\langle Q^{2}\right\rangle_{\tau}^{c}=\left(h \nu_{0}\right)^{2} \mathcal{T}_{\tau}(J)$ $\left[f_{1}\left(1-f_{2}\right)+f_{2}\left(1-f_{1}\right)\right]=\left(h \nu_{0}\right)^{2} \operatorname{coth}\left(\frac{\Delta \beta h \nu_{0}}{2}\right) \mathcal{T}_{\tau}(J)\left(f_{2}-f_{1}\right) \geqslant$ $\frac{2}{\Delta \beta} h v_{0} \mathcal{T}_{\tau}(J)\left(f_{2}-f_{1}\right)=\frac{2}{\Delta \beta}\langle Q\rangle_{\tau}$, proving that the S-TUR is satisfied even far from equilibrium. 